\title{
An Application of Shadow Systems to Mahler's Conjecture
}

\author{
Matthieu Fradelizi • Mathieu Meyer • \\ Artem Zvavitch
}

Received: 4 November 2011 / Revised: 18 June 2012 / Accepted: 18 June 2012 /

Published online: 6 July 2012

(C) Springer Science+Business Media, LLC 2012

\begin{abstract}
We elaborate on the use of shadow systems to prove a particular case of the conjectured lower bound of the volume product $\mathcal{P}(K)=\min _{z \in \operatorname{int}(K)}|K|\left|K^{z}\right|$, where $K \subset \mathbb{R}^{n}$ is a convex body and $K^{z}=\left\{y \in \mathbb{R}^{n}:(y-z) \cdot(x-z) \leq 1\right.$ for all $\left.x \in K\right\}$ is the polar body of $K$ with respect to the center of polarity $z$. In particular, we show that if $K \subset \mathbb{R}^{3}$ is the convex hull of two 2-dimensional convex bodies, then $\mathcal{P}(K) \geq$ $\mathcal{P}\left(\Delta^{3}\right)$, where $\Delta^{3}$ is a 3-dimensional simplex, thus confirming the 3-dimensional case of Mahler conjecture, for this class of bodies. A similar result is provided for the symmetric case, where we prove that if $K \subset \mathbb{R}^{3}$ is symmetric and the convex hull of two 2-dimensional convex bodies, then $\mathcal{P}(K) \geq \mathcal{P}\left(B_{\infty}^{3}\right)$, where $B_{\infty}^{3}$ is the unit cube.
\end{abstract}

Mathematics Subject Classification (2000) 52A40 · 53A15 · 52B10

Keywords Convex bodies · Convex polytopes $\cdot$ Polar bodies $\cdot$ Santaló point · Volume product $\cdot$ Shadow systems

M. Fradelizi · M. Meyer

Laboratoire d'Analyse et de Mathématiques Appliquées (UMR 8050), Université Paris-Est Marne-la-Vallée, Cité Descartes 5, Bd Descartes, Champs-sur-Marne 77454, Marne-la-Vallée Cedex 2, France

M. Fradelizi

e-mail: Matthieu.Fradelizi@univ-mlv.fr

M. Meyer

e-mail: Mathieu.Meyer@univ-mlv.fr

A. Zvavitch (凶)

Department of Mathematical Sciences, Kent State University, Kent, OH 44242, USA

e-mail: zvavitch@math.kent.edu 


\section{Introduction and Preliminaries}

As usual, we denote by $x \cdot y$ the inner product of two vectors $x, y \in \mathbb{R}^{n}$ and by $|x|$ the length of vector $x \in \mathbb{R}^{n}$. For two non-empty subsets $K, L \subset \mathbb{R}^{n}$ we define their Hausdorff distance $d_{H}(K, L)$ by

$$
d_{H}(K, L)=\max \left\{\sup _{x \in K} \inf _{y \in L}|x-y|, \sup _{y \in L} \inf _{x \in K}|x-y|\right\} .
$$

A convex body is a compact convex subset of $\mathbb{R}^{n}$ with non-empty interior and $\mathcal{K}_{n}$ is the set of all convex bodies in $\mathbb{R}^{n}$ endowed with the Hausdorff metric. We say that a set $K$ is symmetric if it is centrally symmetric with center at the origin, i.e. $K=-K$.

We write $|A|$ for the $k$-dimensional Lebesgue measure (volume) of a measurable set $A \subset \mathbb{R}^{n}$, where $k=1, \ldots, n$ is the dimension of the minimal flat containing $A$. We denote by $\overline{\operatorname{conv}}(A)$ the closed convex hull of a set $A \subset \mathbb{R}^{n}$, and $\overline{\operatorname{conv}}(A, B, C, \ldots)$ the closed convex hull of $A \cup B \cup C, \ldots$ For $a, b \in \mathbb{R}^{n}$, we denote $[a, b]$ the segment joining $a$ to $b:[a, b]=\{(1-t) a+t b: t \in[0,1]\}$. If $K \in \mathcal{K}_{n}$, we denote by $\mathcal{E}(K)$ the set of its extreme points. By Caratheodory's theorem, one has $K=\overline{\operatorname{conv}}(\mathcal{E}(K))$. We recommend [9] and [31] as a general references for convex bodies and polytopes and their properties.

By int $(K)$ we denote the interior of $K$. If $K$ is a convex body in $\mathbb{R}^{n}$ and $z \in \operatorname{int}(K)$ the polar body $K^{z}$ of $K$ with the center of polarity $z$ is defined by

$$
K^{z}=\left\{y \in \mathbb{R}^{n}:(y-z) \cdot(x-z) \leq 1 \text { for all } x \in K\right\} .
$$

If the center of polarity is taken to be the origin, we denote by $K^{\circ}$ the polar body of $K$, thus $K^{z}=(K-z)^{\circ}+z$. The bipolar theorem says that $\left(K^{z}\right)^{z}=K$, for $z \in \operatorname{int}(K)$ (see [9], p. 47).

A well known result of Santaló [29] (see also [31, p. 419]) states that in every convex body $K$ in $\mathbb{R}^{n}$, there exists a unique point $s(K)$, called the Santaló point of $K$, such that

$$
\left|K^{s(K)}\right|=\min _{z \in \operatorname{int}(K)}\left|K^{z}\right| .
$$

The volume product of $K$ is defined by

$$
\mathcal{P}(K)=\inf \left\{|K|\left|K^{z}\right|: z \in \operatorname{int}(K)\right\}=|K|\left|K^{s(K)}\right| .
$$

The volume product is affinely invariant, that is, $\mathcal{P}(A(K))=\mathcal{P}(K)$ for every affine isomorphism $A: \mathbb{R}^{n} \rightarrow \mathbb{R}^{n}$. Observe that if we denote $L=K^{s(K)}$ then

$$
\mathcal{P}\left(K^{s(K)}\right)=|L|\left|L^{s(L)}\right| \leq|L|\left|L^{s(K)}\right|=\left|K^{s(K)}\right||K|=\mathcal{P}(K) .
$$

The set of all convex bodies in $\mathbb{R}^{n}$ is compact with respect to the Banach-Mazur distance and $K \mapsto \mathcal{P}(K)$ is continuous (see Lemma 3, below), so that it is natural to ask for a maximal and minimal values of $\mathcal{P}(K)$. The Blaschke-Santaló inequality states that

$$
\mathcal{P}(K) \leq \mathcal{P}\left(B_{2}^{n}\right)
$$


where $B_{2}^{n}$ is the Euclidean unit ball. The equality in the above inequality is possible only for ellipsoids ([23, 29]; see [18] or also [20] for a simple proof of both the inequality and the case of equality).

The main focus of this paper is the conjecture about minimality of $\mathcal{P}(K)$, often called Mahler's conjecture [13, 14], which states that, for every convex body $K$ in $\mathbb{R}^{n}$,

$$
\mathcal{P}(K) \geq \mathcal{P}\left(\Delta^{n}\right)=\frac{(n+1)^{n+1}}{(n !)^{2}},
$$

where $\Delta^{n}$ is an $n$-dimensional simplex. It is also conjectured that equality in (1) is attained only if $K$ is a simplex.

We shall also pay a special attention to the symmetric case of Mahler conjecture which states that for every convex symmetric body $K \subset \mathbb{R}^{n}$ :

$$
\mathcal{P}(K) \geq \mathcal{P}\left(B_{1}^{n}\right)=\mathcal{P}\left(B_{\infty}^{n}\right)=\frac{4^{n}}{n !},
$$

where $B_{1}^{n}$ and $B_{\infty}^{n}$ are cross-polytope and its dual cube, respectively.

The inequalities (1) and (2) for $n=2$ were proved by Mahler [13] with the case of equality proved by Meyer [17] in the general case and by Reisner [25] in the symmetric case. Other cases, like e.g. the bodies of revolution, were treated in [19]. Several special cases of the (mostly) symmetric case of the conjecture can be found in $[4,6,7,11,16,22,25-27,30]$. Not many special cases in which (1) is true seem to be known, one such is the case of convex bodies having hyperplane symmetries which fix only one common point [1]; another one is the $n$ dimensional polytopes with at most $n+3$ vertices (or facets) proved in [20]. The proof of this last result is based on the method of shadow systems. We shall here elaborate on this method, applying it alternatively to a body and its polar.

Observe that an isomorphic version of reverse Santaló inequality was proved by Bourgain and Milman [2], see also [24, p. 100]:

$$
\mathcal{P}(K) \geq c^{n} \mathcal{P}\left(B_{2}^{n}\right),
$$

where $c$ is a positive constant; Kuperberg [12] gave a new proof of this result with a better constant (see also [5, 21] for different proofs of the inequality).

It is conjectured that in the centrally symmetric case, the convex bodies which are minimal for the volume product are the unit balls of Lima spaces, i.e. the finite dimensional normed spaces with the 3-2 intersection property: every three translates of the unit ball which intersect 2 by 2 , actually intersect. As normed spaces, Lima spaces are characterized by the fact that they can be decomposed into $\ell_{\infty}$ or $\ell_{1}$ sums of normed spaces of smaller dimension satisfying the same decomposition property [10]. In the special cases of convex bodies symmetric with respect to $n$ independent hyperplanes, it was proved in $[16,26]$ that the unit ball of Lima spaces are the minimizers of $\mathcal{P}(K)$. The unit ball of Lima spaces have the special property (which does not characterize them): any facet of their unit ball contains half of the extreme points so that they are the convex hull of any of two of their opposite facets (see [10]). The purpose of this paper was originally to try to prove that unit balls of finite dimensional spaces which 
have this last property satisfy Mahler conjecture. We proved it in dimension 3 . Then we realized that our method of proof can be used for a much more general result: if a convex body in $\mathbb{R}^{3}$ is the convex hull of two of its hyperplane sections, then it satisfies Mahler's conjecture (Theorem 1).

Thus the main goal of this paper is to prove the following special cases of inequalities (1) and (2):

Theorem 1 Consider two different planes $H_{1}$ and $H_{2}$ in $\mathbb{R}^{3}$ and a convex body $K$ in $\mathbb{R}^{3}$ such that

$$
K=\overline{\operatorname{conv}}\left(K \cap H_{1}, K \cap H_{2}\right) .
$$

Then

$$
\mathcal{P}(K) \geq \mathcal{P}\left(\Delta_{3}\right)=\frac{64}{9} .
$$

If, moreover, $K$ is centrally symmetric then

$$
\mathcal{P}(K) \geq \mathcal{P}\left(B_{\infty}^{3}\right)=\frac{32}{3} .
$$

Remark 1 Notice that if $K=\overline{\operatorname{conv}}\left(K \cap H_{1}, K \cap H_{2}\right)$ is centrally symmetric then

- either $H_{1}$ is parallel to $H_{2}$, the central symmetry maps $H_{1}$ onto $H_{2}$ and $K \cap H_{1}$ onto $\mathrm{K} \cap \mathrm{H}_{2}$.

- or $H_{1}$ and $H_{2}$ intersect and $K \cap H_{1}$ and $K \cap H_{2}$ are centrally symmetric.

From Theorem 1 and the first case of Remark 1 we deduce the following corollary.

Corollary 1 Let $K$ be a centrally symmetric convex body in $\mathbb{R}^{3}$ whose extreme points lie into two parallel planes. Then

$$
\mathcal{P}(L) \geq \mathcal{P}\left(B_{\infty}^{3}\right)=\frac{32}{3} .
$$

We would like to note that most of the tools presented in Sect. 2 are stated and proved in dimension $n \geq 2$. Still, unfortunately, the proof of Theorem 1, in Sect. 3, requires to use the special geometrical structure of 3-dimensional polytopes as well as the assumption that the Mahler conjecture is true in $\mathbb{R}^{n-1}$, thus restricting to the case $n=3$.

\section{The Tools}

As the main tool in the proof of Theorem 1, we will use shadow systems of convex sets: a shadow system of convex sets along a direction $\theta \in \mathbb{S}^{n-1}$ is a family of convex sets $L_{t} \in \mathbb{R}^{n}$ which are defined by

$$
L_{t}=\overline{\operatorname{conv}}\{x+\alpha(x) t \theta: x \in B\},
$$


where $B \subset \mathbb{R}^{n}$ is a bounded set, called the basis of the shadow system, $\alpha: B \rightarrow \mathbb{R}$ is a bounded function, called the speed of the shadow system and $t$ belongs to an open interval in $\mathbb{R}$. We say that a shadow system is non-degenerate, if all the convex sets $L_{t}$ have non-empty interior. The shadow systems were introduced by Rogers and Shephard [28]. Campi and Gronchi [3] proved that if $L_{t}$ is a symmetric shadow system then $t \mapsto\left|L_{t}^{\circ}\right|^{-1}$ is a convex function of $t$. In [20], Reisner and the second author generalized this result to the non-symmetric case and studied the equality case. The following proposition is the key tool in the proof of Theorem 1:

Proposition 1 [20] Let $L_{t}, t \in[-a, a]$, be a non-degenerate shadow system in $\mathbb{R}^{n}$, with direction $\theta \in \mathbb{S}^{n-1}$, then $t \mapsto\left|L_{t}^{s\left(L_{t}\right)}\right|^{-1}$ is a convex function on $[-a, a]$.

If, moreover, $t \mapsto\left|L_{t}\right|$ is affine on $[-a, a]$ and $t \mapsto \mathcal{P}\left(L_{t}\right)$ is constant on $[-a, a]$, then there exist $w \in \mathbb{R}^{n}$ and $\alpha \in \mathbb{R}$, such that for every $t \in[-a, a]$, one has $L_{t}=$ $A_{t}\left(L_{0}\right)$, where $A_{t}: \mathbb{R}^{n} \rightarrow \mathbb{R}^{n}$ is the affine map defined by

$$
A_{t}(x)=x+t(w \cdot x+\alpha) \theta \text {. }
$$

The following corollary is a variation of the above proposition that we shall need.

Corollary 2 Let $L_{t}, t \in[-a, a]$, be a non-degenerate shadow system in $\mathbb{R}^{n}$ such that $t \mapsto\left|L_{t}\right|$ is affine on $[-a, a]$. Then

(1) $t \mapsto \mathcal{P}\left(L_{t}\right)$ is quasi-concave on $[-a, a]$, i.e. for every interval $[c, d] \subset[-a, a]$ one has $\min _{[c, d]} \mathcal{P}\left(L_{t}\right)=\min \left\{\mathcal{P}\left(L_{c}\right), \mathcal{P}\left(L_{d}\right)\right\}$;

(2) if for some $t_{0} \in(-c, c) \subset[-a, a]$, one has $\mathcal{P}\left(L_{t_{0}}\right)=\min _{t \in[-c, c]} \mathcal{P}\left(L_{t}\right)$, then either $\mathcal{P}\left(L_{t}\right)=\mathcal{P}\left(L_{t_{0}}\right)$ for every $t \in\left[t_{0}, c\right]$, or $\mathcal{P}\left(L_{t}\right)=\mathcal{P}\left(L_{t_{0}}\right)$ for every $t \in\left[-c, t_{0}\right]$.

Proof of Corollary 2 (1) The volume product of $L_{t}$ is the quotient of the affine positive function $f(t):=\left|L_{t}\right|$ by the convex (from Proposition 1) positive function $g(t):=\left|L_{t}^{s\left(L_{t}\right)}\right|^{-1}$. Such a quotient is quasi-concave because for every interval $[c, d] \subset[a, b]$ and any $\lambda \in[0,1]$ one has

$$
\frac{f((1-\lambda) c+\lambda d)}{g((1-\lambda) c+\lambda d)} \geq \frac{(1-\lambda) f(c)+\lambda f(d)}{(1-\lambda) g(c)+\lambda g(d)} \geq \min \left(\frac{f(c)}{g(c)}, \frac{f(d)}{g(d)}\right) .
$$

(2) Notice first that if $h$ is a quasi-concave function on some interval $[c, d]$ and $\min _{[c, d]} h=h\left(t_{0}\right)$, for some $t_{0} \in(c, d)$, then $h$ is constant on either $\left[c, t_{0}\right]$ or on $\left[t_{0}, d\right]$. Indeed if $h$ was not constant on any of these intervals, there would exist $u \in\left[c, t_{0}\right)$ and $v \in\left(t_{0}, d\right]$ such that $h(u)>h\left(t_{0}\right)$ and $h(v)>h\left(t_{0}\right)$. But since $t_{0} \in(u, v)$ this contradicts the fact that $\min _{[u, v]} h=\min \{h(u), h(v)\}$. It follows that $h$ is constant on at least one of these intervals.

We need now to define a particular form of shadow system:

Definition 1 Consider $K \in \mathcal{K}_{n}, x \in \mathcal{E}(K)$ and $\theta \in \mathbb{S}^{n-1}$. Let $R_{x}(K)=\overline{\operatorname{conv}}(\mathcal{E}(K) \backslash$ $\{x\})$. Define $x_{t}=x+t \theta$ and $K_{t}=\overline{\operatorname{conv}}\left(R_{x}(K), x_{t}\right)$. Then $t \mapsto K_{t}$ is called the 
shadow movement of $K$ based on $x$ with direction $\theta$. Such a shadow movement is actually a shadow system, with $B=\mathcal{E}(K)$ and speed $\alpha: \mathcal{E}(K) \rightarrow \mathbb{R}$ defined by $\alpha(y)=0$ if $y \neq x$ and $\alpha(x)=1$. Observe that one has of course $K_{0}=K$. We say that a shadow movement $\left(K_{t}\right)$ is volume affine on $[-c, c]$ if $t \mapsto\left|K_{t}\right|$ is affine on $[-c, c]$.

We shall need the following easy lemma (see, for example, Lemma 11 in [20]), where we use the fact that inequalities (1) and (2) hold in dimension 2, by the original Mahler's result.

Lemma 1 Let $H$ be a hyperplane in $\mathbb{R}^{n}, B$ be a convex body in $H, x \notin H$.

(1) Let $C$ be the cone with apex $x$ and basis $B$, i.e. $C=\overline{\operatorname{conv}}(B,\{x\})$. Then one has $\mathcal{P}(C) \geq \frac{(n+1)^{n+1}}{n^{n+2}} \mathcal{P}(B)$. In particular, if $n=3$, then $\mathcal{P}(C) \geq \mathcal{P}\left(\Delta^{3}\right)$.

(2) If $B$ is symmetric, let $D=\overline{\operatorname{conv}}(B,\{x\},\{-x\})$ be a double cone with basis $B$. Then one has $\mathcal{P}(D) \geq \frac{4}{n} \mathcal{P}(B)$ and in particular, if $n=3$, then $\mathcal{P}(D) \geq \mathcal{P}\left(B_{1}^{3}\right)=$ $\mathcal{P}\left(B_{\infty}^{3}\right)$.

Lemma 2 Let $t \mapsto K_{t}, t \in[-c, c]$ be a volume affine shadow movement of a convex body $K \subset \mathbb{R}^{n}$ based on $x \in \mathcal{E}(K)$ with direction $\theta \in \mathbb{S}^{n-1}$. Suppose, moreover, that

$$
\mathcal{P}(K)=\min _{t \in[-c, c]} \mathcal{P}\left(K_{t}\right) .
$$

Then $\mathcal{E}(K) \backslash\{x\}$ is contained in a hyperplane, $K$ is a cone with apex $x$ and basis $\overline{\operatorname{conv}}(\mathcal{E}(K) \backslash\{x\})$ and

$$
\mathcal{P}(K) \geq \frac{(n+1)^{n+1}}{n^{n+2}} \min _{M \in \mathcal{K}_{n-1}} \mathcal{P}(M) .
$$

Proof Let $R_{x}(K)=\overline{\operatorname{conv}}(\mathcal{E}(K) \backslash\{x\})$. Observe that $x \notin R_{x}(K)$. Otherwise, one would have $K \subset K_{t}$, hence $|K| \leq\left|K_{t}\right|$ for every $t$, since $t \mapsto\left|K_{t}\right|$ is affine on interval $[-c, c]$, it may have minimum a $t=0$ only if it is a constant on $[-c, c]$. Thus $K=K_{t}$, which implies that $x_{t} \in K$ for every $t \in[-c, c]$, which contradicts the extremality of $x \in K$.

It follows that $d\left(x, R_{x}(K)\right)>0$, hence there exist $\gamma>0$ and $c^{\prime}>0$ such that $d\left(x+t \theta, R_{x}(K)\right)>\gamma$ for all $t \in\left[-c^{\prime}, c^{\prime}\right]$. Thus, for all $t \in\left[-c^{\prime}, c^{\prime}\right], x+t \theta$ is an extreme point of $K_{t}$. Decreasing $c$, we may suppose that $c^{\prime}=c$.

By the second part of Corollary 2, we may suppose also that $\mathcal{P}\left(K_{t}\right)=\mathcal{P}(K)$ for every $t \in[0, c]$. Let $d:=\frac{c}{2}$ and $L=K_{d}$. We define the shadow movement of $L$ based on $x+d \theta$ with direction $\theta$. We notice that for $s \in[-d, d]$, one has $L_{s}=K_{d+s}$. Of course the shadow movement $\left(L_{s}\right)$ is also volume affine on $[-d, d]$ and satisfy $s \mapsto \mathcal{P}\left(L_{s}\right)$ is constant on $[-d, d]$.

By Proposition 1 , there exist $\alpha \in \mathbb{R}$ and $w \in \mathbb{R}^{n}$ such that for all $s \in[-d, d]$, one has $L_{s}=A_{s}(L)$, where $A_{s}: \mathbb{R}^{n} \rightarrow \mathbb{R}^{n}$ is the affine isomorphism defined by $A_{s}(z)=z+s(z \cdot w+\alpha) \theta$.

Now, since $A_{s}$ is affine, it maps the extreme points of $L$ onto the extreme points of $L_{s},|s| \leq d$. Thus $A_{s}(x+d \theta)$ is an extreme point of $L_{s}$, that is, $A_{s}(x+d \theta) \in$ $R_{x}(K) \cup\{x+(d+s) \theta\}$. But for $|s|$ small enough $A_{s}(x+d \theta)$ is close to $x+d \theta$, 
and thus far from $R_{x}(K)$. It follows that $A_{s}(x+d \theta)=x+(d+s) \theta$ for all $s$ small enough and thus also $A_{s}\left(R_{x}(K)\right)=R_{x}(K)$. Then $A_{s}$ is an isometry (with respect to the Euclidean scalar product), when $R_{x}(K)$ has non-empty interior. From the special form of $A_{s}$ this can only happen if $A_{s}$ is the identity. But $A_{s}(x+d \theta)=x+(d+s) \theta \neq$ $x+d \theta$, for $s \neq 0$, thus we get a contradiction and $R_{x}(K)$ must have an empty interior. Being convex $R_{x}(K)$, must be contained in a hyperplane. Thus $K=\overline{\operatorname{conv}}\left(R_{x}(K), x\right)$ is a cone with apex $x$ and basis $R_{x}(K)$. The result follows from Lemma 1 .

For sake of completeness, we prove the following classical result, which is needed to restrict the proof of Theorem 1 to the case of polytopes:

Lemma $3 K \mapsto \mathcal{P}(K)$ is a continuous function on $\mathcal{K}_{n}$.

Proof Let $K_{m}, m \geq 0$ and $K$ be compact convex bodies such that $K_{m} \rightarrow K$. By John's Theorem (see, for example, [24], p. 33) there is an affine isomorphism $A: \mathbb{R}^{n} \rightarrow \mathbb{R}^{n}$ such that $B_{2}^{n} \subset L:=A(K) \subset n B_{2}^{n}$. If $L_{m}:=A\left(K_{m}\right)$, one has still $L_{m} \rightarrow L$. There exist constants $c, d>0$ and $M \geq 0$ such that for any $m \geq M$, one has $c B_{2}^{n} \subset L_{m} \subset d B_{2}^{n}$ and $c B_{2}^{n} \subset L \subset d B_{2}^{n}$. Since $L_{m} \rightarrow L$, for every $\varepsilon>0$ there exists $M^{\prime}$ such that $L_{m} \subset L+\varepsilon B_{2}^{n}$ and $L \subset L_{m}+\varepsilon B_{2}^{n}$, for every $m \geq M^{\prime}$. It follows that $L_{m} \subset(1+\eta) L$ and $L \subset(1+\eta) L_{m}$, for $m \geq \max \left\{M, M^{\prime}\right\}$ and $\eta=\frac{\varepsilon}{c}$. Thus $L^{s\left(L_{m}\right)} \subset(1+\eta) L_{m}^{s\left(L_{m}\right)}$ and

$$
\mathcal{P}(L)=|L|\left|L^{s(L)}\right| \leq|L|\left|L^{s\left(L_{m}\right)}\right| \leq(1+\eta)^{2 n}\left|L_{m}\right|\left|L_{m}^{s\left(L_{m}\right)}\right|=(1+\eta)^{2 n} \mathcal{P}\left(L_{m}\right),
$$

for $m \geq M$. Similarly, $L_{m}^{s(L)} \subset(1+\eta) L^{s(L)}$ and

$$
\mathcal{P}\left(L_{m}\right)=\left|L_{m}\right|\left|L_{m}^{s\left(L_{m}\right)}\right| \leq\left|L_{m}\right|\left|L_{m}^{s(L)}\right| \leq(1+\eta)^{2 n}|L|\left|L^{s(L)}\right|=(1+\eta)^{2 n} \mathcal{P}(L) .
$$

Since $\mathcal{P}\left(L_{m}\right)=\mathcal{P}\left(A\left(K_{m}\right)\right)=\mathcal{P}\left(K_{m}\right)$ and $\mathcal{P}(L)=\mathcal{P}(A(K))=\mathcal{P}(K)$, we can conclude.

Proposition 2 Suppose that $\mathcal{C}$ is a closed subset of $\mathcal{K}_{n}$ such that for any $K \in \mathcal{C}$, any $z \in \operatorname{int}(K)$, any $y \in \operatorname{int}\left(K^{z}\right)$ and any affine isomorphism $A: \mathbb{R}^{n} \rightarrow \mathbb{R}^{n}$, one has $\left(K^{z}\right)^{y} \in \mathcal{C}$ and $A(K) \in \mathcal{C}$. Then:

(1) There exists $Q \in \mathcal{C}$ such that $\mathcal{P}(Q)=\min _{P \in \mathcal{C}} \mathcal{P}(P)$.

(2) Moreover, if one of the following hypothesis holds:

(i) there exists a volume affine shadow movement $\left(Q_{t}\right)_{t \in[-c, c]}$ of $Q$ with basis $x \in \mathcal{E}(Q)$ such that $Q_{t} \in \mathcal{C}$ for $t \in[-c, c]$,

(ii) there exists a volume affine shadow movement $\left(L_{t}\right)_{t \in[-c, c]}$ of $L=Q^{s(Q)}$ with basis $y \in \mathcal{E}(L), t \in[-c, c]$ such that $\left(L_{t}\right)^{s(L)} \in \mathcal{C}$ for $t \in[-c, c]$,

then for every $P \in \mathcal{C}$, one has $\mathcal{P}(P) \geq \frac{(n+1)^{n+1}}{n^{n+2}} \min _{M \in \mathcal{K}_{n-1}} \mathcal{P}(M)$.

Proof Let $\gamma=\inf _{K \in \mathcal{C}} \mathcal{P}(K)$. Then there exists a sequence $\left(P_{m}\right) \in \mathcal{C}$ such that $\mathcal{P}\left(P_{m}\right) \rightarrow \gamma$. Applying John's Theorem, one can find a sequence $\left\{A_{m}\right\}$ of affine isomorphisms such that

$$
B_{2}^{n} \subset Q_{m}:=A_{m}\left(P_{m}\right) \subset n B_{2}^{n}, \quad \text { for all } m \in \mathbb{N} .
$$


But $Q_{m} \in \mathcal{C}$, from the assumption of Proposition 2 and $\mathcal{P}\left(Q_{m}\right)=\mathcal{P}\left(P_{m}\right) \rightarrow \gamma$. The set $\left\{K \in \mathcal{K}_{n}: B_{2}^{n} \subset K \subset n B_{2}^{n}\right\}$ is compact with respect to the Hausdorff distance. Thus we may assume that $\left\{Q_{m}\right\}_{m}$ converges to some $Q \in \mathcal{K}_{n}$. Since $\mathcal{C}$ is closed, $Q \in \mathcal{C}$ and since, by Lemma $3, K \mapsto \mathcal{P}(K)$ is continuous on $\mathcal{K}_{n}$, we get $\mathcal{P}(Q)=\gamma$. Under hypothesis (i), the result follows immediately from Lemma 2 . Now, under hypothesis (ii), with the above notation, we get

$$
M_{t}=\left(L_{t}\right)^{s(L)} \in \mathcal{C}, \quad L_{t}=\left(M_{t}\right)^{s(L)} \quad \text { and } \quad L_{t}^{s\left(L_{t}\right)}=\left(\left(M_{t}\right)^{s(L)}\right)^{s\left(L_{t}\right)},
$$

for small enough $|t|$. It follows that $L_{t}^{s\left(L_{t}\right)} \in \mathcal{C}$. Thus

$$
\mathcal{P}(Q) \leq \mathcal{P}\left(L_{t}^{s\left(L_{t}\right)}\right) \leq \mathcal{P}\left(L_{t}\right)
$$

Applying this to $t=0$ and since $L_{0}=L=Q^{s(Q)}$, one has also

$$
\mathcal{P}(Q) \leq \mathcal{P}\left(L_{0}\right)=\mathcal{P}(L)=\mathcal{P}\left(Q^{s(Q)}\right) \leq \mathcal{P}(Q) .
$$

Thus $L_{t}$ is a volume affine shadow movement whose volume product is minimized at 0 . From Lemma 2, we conclude that $L=Q^{s(Q)}$ is a cone and for every $P \in \mathcal{C}$ one has

$$
\mathcal{P}(P) \geq \mathcal{P}(Q) \geq \mathcal{P}\left(Q^{s(Q)}\right) \geq \frac{(n+1)^{n+1}}{n^{n+2}} \min _{M \in \mathcal{K}_{n-1}} \mathcal{P}(M) .
$$

Definition 2 For $N \geq n+1$, we define $\mathcal{P}_{N}$ to be the set of all convex polytopes in $\mathbb{R}^{n}$ with non-empty interior, having at most $N$ vertices, $\mathcal{C}_{N}$ to be the set of all polytopes in $\mathcal{P}_{N}$ which are the convex hull of two of their hyperplane sections and $\mathcal{D}_{N}$ to be the set of all polytopes in $\mathcal{P}_{N}$ which are the convex hull of two of their parallel facets.

Lemma 4 Let $N \geq n+1$. Then $\mathcal{P}_{N}, \mathcal{C}_{N}$ and $\mathcal{D}_{N}$ are closed subsets of $\mathcal{K}_{n}$. Moreover, if $P$ is a polytope in $\mathbb{R}^{n}, z \in \operatorname{int}(P), y \in \operatorname{int}\left(P^{z}\right)$ and $A: \mathbb{R}^{n} \rightarrow \mathbb{R}^{n}$ is an affine isomorphism and $P \in \mathcal{P}_{N}$ (resp. $\left.\mathcal{C}_{N}, \mathcal{D}_{N}\right)$, then so do $\left(P^{z}\right)^{y}$ and $A(P)$.

Proof We use standard arguments of compactness for the fact that these classes are closed. It also follows from the definition that the classes are stable under affine isomorphisms. Next we observe that if $K \in \mathcal{K}_{n}, z \in \operatorname{int}(K)$ and $y \in \operatorname{int}\left(K^{z}\right)$, then

$$
\left(K^{z}\right)^{y}=\left\{\frac{x-z}{1-(x-z) \cdot(y-z)}+y: x \in K\right\} .
$$

The above formula follows immediately by applying polarity with respect to $y$ to both sets. To show that $\left(P^{z}\right)^{y}$ is in the same class as $P$, we define $F: K \rightarrow\left(K^{z}\right)^{y}$ by

$$
F(x)=\frac{x-z}{1-(x-z) \cdot(y-z)}+y .
$$

We note that $F$ is a bijection preserving segments. Indeed, let $x_{1}, x_{2} \in K$ and $\lambda \in[0,1]$. We set

$$
\mu=\frac{\lambda\left(1-\left(x_{2}-z\right) \cdot(y-z)\right)}{(1-\lambda)\left(1-\left(x_{1}-z\right) \cdot(y-z)\right)+\lambda\left(1-\left(x_{2}-z\right) \cdot(y-z)\right)} .
$$


Then $F\left((1-\lambda) x_{1}+\lambda x_{2}\right)=(1-\mu) F\left(x_{1}\right)+\mu F\left(x_{2}\right)$. Hence, $F\left(\left[x_{1}, x_{2}\right]\right)=$ $\left[F\left(x_{1}\right), F\left(x_{2}\right)\right]$ and $F$ maps extreme points into extreme points and hyperplanes to hyperplanes, hence preserves $\mathcal{P}_{N}, \mathcal{C}_{N}$ and $\mathcal{D}_{N}$.

Lemma 5 Let $P$ be a convex polytope in $\mathbb{R}^{n}$ and $x$ be a vertex of $P$. Suppose that for some $0 \leq k \leq n-1$, all but $k$ facets $F_{1}, \ldots, F_{k}$ of $P$ containing $x$ are $(n-1)$ dimensional cones with apex $x$. Let $H_{1}, \ldots, H_{k}$ be their supporting hyperplanes, $E=$ $\bigcap_{i=1}^{k} H_{i}$ and $Q=\overline{\operatorname{conv}}(\mathcal{E}(P) \backslash\{x\})$. For $y \in \mathbb{R}^{n}$, let $P(y)=\overline{\operatorname{conv}}(Q, y)$. Then:

(1) $P(x)=P, \operatorname{dim}(E) \geq n-k$ and $y \mapsto|P(y)|$ is affine on a neighborhood of $x$ in $E$.

(2) For any direction $\theta \in \mathbb{S}^{n-1}$ parallel to $E$, there exist $c>0$ and a shadow movement $\left(P_{t}\right)$ of $P$, with basis $x$ and direction $\theta$, which is volume affine on $[-c, c]$.

Proof Without loss of generality we may assume that $0 \in \operatorname{int}(Q)$. Let $\left(G_{m}\right), 1 \leq m \leq$ $M$, be the facets of $Q$, with supporting hyperplanes $\left\{z: z \cdot v_{m}=h_{m}\right\}, v_{m} \in \mathbb{S}^{n-1}$, $h_{m}>0$ so that

$$
Q=\bigcap_{m=1}^{M}\left\{z: z \cdot v_{m} \leq h_{m}\right\} \quad \text { and } \quad|Q|=\frac{1}{n} \sum_{m=1}^{M} h_{m}\left|G_{m}\right| .
$$

Then

$$
\begin{aligned}
|P(y)| & =|Q|+\frac{1}{n} \sum_{m=1}^{M}\left(y \cdot v_{m}-h_{m}\right)_{+}\left|G_{m}\right| \\
& =\frac{1}{n} \sum_{m=1}^{M} \max \left\{y \cdot v_{m}, h_{m}\right\}\left|G_{m}\right| .
\end{aligned}
$$

Observe that if $1 \leq m \leq k, F_{m} \cap Q$ is a facet of $Q$. With a reordering, we may suppose that $G_{m}=F_{m} \cap Q$, for $1 \leq m \leq k$. Moreover, for $k+1 \leq m \leq M$, the supporting hyperplane $H_{m}$ of $G_{m}$ does not contain $x$ (indeed if $x \cdot v_{m}=h_{m}$, then $H_{m} \cap P$ is a facet of $P$ which is not a cone with apex $x$ ). Thus,

$$
|P(y)|=\frac{1}{n} \sum_{m=k+1}^{M} \max \left\{y \cdot v_{m}, h_{m}\right\}\left|G_{m}\right|+\frac{1}{n} \sum_{m=1}^{k} h_{m}\left|G_{m}\right|, \quad \text { for } y \in E,
$$

and $|P(y)|$ is affine function in the neighborhood $V(x) \subset E$ defined by

$$
V(x)=\left\{y \in E:\left(y \cdot v_{m}-h_{m}\right) \cdot\left(x \cdot v_{m}-h_{m}\right)>0: k+1 \leq m \leq M\right\} .
$$

\section{Proof of Theorem 1}

It is enough to prove Theorem 1 for the case of polytopes. The result for general bodies follows from Lemma 3 and a standard approximation procedure (see, for example, [8, 32]). 
We first prove the general case and we shall treat the symmetric case afterwards. Let $N \geq 4$, and $\mathcal{C}_{N}$ be the set of convex polytopes $P$ in $\mathbb{R}^{3}$, with not more than $N$ vertices, such that for some different hyperplanes $H_{1}$ and $H_{2}$ one has $P=\overline{\operatorname{conv}}(P \cap$ $H_{1}, P \cap H_{2}$ ). We notice that this class is closed in the Hausdorff metric, thus by Proposition 2 and Lemma 4, there exists $Q \in \mathcal{C}_{N}$ such that

$$
\mathcal{P}(Q)=\min _{P \in \mathcal{C}_{N}} \mathcal{P}(P) .
$$

We shall prove that $\mathcal{P}(Q) \geq \mathcal{P}\left(\Delta^{3}\right)$. To do this we shall distinguish three cases according to the dimension of $H_{1} \cap H_{2} \cap Q$ :

Case 1: $H_{1} \cap H_{2} \cap Q$ is a segment $[p, m] \quad$ We shall consider 3 subcases.

Subcase 1.i: The point $m$ is in the interior of a facet of $Q$. Then $m=\left[a_{1}, b_{1}\right] \cap$ $\left[a_{2}, b_{2}\right]$, where $\left[a_{i}, b_{i}\right]$ are edges of $H_{i} \cap Q$, with $m \neq a_{i}, b_{i}, i=1,2$. Thus $F:=$ $\overline{\operatorname{conv}}\left(a_{1}, b_{1}, a_{2}, b_{2}\right)$ is a face of $Q$ containing $m$. Next we shall study the edges of $Q$, which are not edges of $F$, but have a common vertex with $F$. More precisely, consider $a_{i}^{\prime}, b_{i}^{\prime} \in H_{i} \cap Q$, such that $a_{i}^{\prime} \notin\left\{a_{i}, b_{i}\right\}, b_{i}^{\prime} \notin\left\{a_{i}, b_{i}\right\}$ are such that $\left[a_{i}^{\prime}, a_{i}\right]$ and $\left[b_{i}^{\prime}, b_{i}\right]$ are edges of $Q_{i}:=Q \cap H_{i}$. If $a_{i}^{\prime}=b_{i}^{\prime}=p$ for $i=1$ or $i=2$, then $Q$ is a cone, and we can conclude applying Lemma 1 . For $i=1,2$, let $m_{i}$ be the intersection of the lines supporting $\left[a_{i}, a_{i}^{\prime}\right]$ and $[p, m]$ and $n_{i}$ be the intersection of the lines supporting $\left[b_{i}, b_{i}^{\prime}\right]$ and $[p, m]$. We denote $\alpha_{i}=\overline{m m_{i}}$ and $\beta_{i}=\overline{m n_{i}}$, the oriented lengths of the segments $\left[m, m_{i}\right]$ and $\left[n, n_{i}\right]$. Observe that $\alpha_{i}, \beta_{i} \neq 0$. We set $\alpha_{i}=+\infty$ or $\beta_{i}=+\infty$ if $\left[a_{i}, a_{i}^{\prime}\right]$ is parallel to $[p, m]$ or $\left[b_{i}, b_{i}^{\prime}\right]$ is parallel to $[p, m]$. We set $\frac{1}{+\infty}=0$.

We shall use the following easy facts:

Fact $1\left(a_{1}, a_{1}^{\prime}, a_{2}, a_{2}^{\prime}\right)$ (resp. $\left.\left(a_{1}, a_{1}^{\prime}, b_{2}, b_{2}^{\prime}\right),\left(b_{1}, b_{1}^{\prime}, a_{2}, a_{2}^{\prime}\right),\left(b_{1}, b_{1}^{\prime}, b_{2}, b_{2}^{\prime}\right)\right)$ are the vertices of a face of $Q$ if and only if $\alpha_{1}=\alpha_{2}$ (resp. $\alpha_{1}=\beta_{2}, \beta_{1}=\alpha_{2}, \beta_{1}=\beta_{2}$ ).

Fact 2 If $\frac{1}{\alpha_{2}}<\frac{1}{\alpha_{1}}$, then $\left(a_{1}, a_{1}^{\prime}, a_{2}\right)$ are the vertices of a triangular face of $Q$ and $\left(a_{2}, a_{2}^{\prime}, a_{1}^{\prime}\right)$ are vertices of another face of $Q$. If $\frac{1}{\alpha_{1}}<\frac{1}{\alpha_{2}}$, then $\left(a_{2}, a_{2}^{\prime}, a_{1}\right)$ are the vertices of a triangular face of $Q$ and $\left(a_{1}, a_{1}^{\prime}, a_{2}^{\prime}\right)$ are vertices of another face of $Q$. A similar statement holds with $\beta_{i}$ instead of $\alpha_{i}, b_{i}$ instead of $a_{i}$ and $b_{i}^{\prime}$ instead of $a_{i}^{\prime}$, for $i=1$ or 2 .

We say that $a_{i}, i=1,2$ is a simple point if $\overline{\operatorname{conv}}\left(a_{i}, a_{i}^{\prime}, a_{j}\right)$ and $\overline{\operatorname{conv}}\left(a_{i}, a_{i}^{\prime}, b_{j}\right)$, $j \neq i$, are side faces of $Q$ and similarly that $b_{i}, i=1,2$ is a simple point if $\overline{\operatorname{conv}}\left(b_{i}, b_{i}^{\prime}, b_{j}\right)$ and $\overline{\operatorname{conv}}\left(b_{i}, b_{i}^{\prime}, a_{j}\right), j \neq i$, are side faces of $Q$. Observe that if $a_{i}$ or $b_{i}, i=1,2$, is simple, then these are the only two faces of $Q$ together with $F$ containing this point.

Fact 3 For $i=1,2, a_{i}$ is a simple point if and only if $\frac{1}{\alpha_{i}}>\max \left(\frac{1}{\beta_{j}}, \frac{1}{\alpha_{j}}\right), j \neq i$, and $b_{i}$ is a simple point if an only if $\frac{1}{\beta_{i}}>\max \left(\frac{1}{\alpha_{j}}, \frac{1}{\beta_{j}}\right), j \neq i$.

Fact 4 Assume that at least one of the points (say $a_{1}$ ) among $a_{i}, b_{i}, i=1,2$, is simple. Thus there are exactly three faces, two of which are triangles, connected to $a_{1}$. We 
define a shadow movement $Q_{t}$ with direction $\left[a_{1}, b_{1}\right]$, based on $a_{1}$. From Lemma 5 , this shadow movement of $Q$ is volume affine on some interval $[-c, c], c>0$. We conclude using the minimality of $Q$ and Lemmas 2 and 4.

Conclusion It follows from Fact 3 and Fact 4 that there is a simple point among $a_{i}, b_{i}, i=1,2$ if and only if

$$
\max \left(\frac{1}{\beta_{1}}, \frac{1}{\alpha_{1}}\right) \neq \max \left(\frac{1}{\beta_{2}}, \frac{1}{\alpha_{2}}\right) .
$$

Suppose now that none of the points $a_{i}, b_{i}, i=1,2$, is simple. Then one has

$$
\max \left(\frac{1}{\beta_{1}}, \frac{1}{\alpha_{1}}\right)=\max \left(\frac{1}{\beta_{2}}, \frac{1}{\alpha_{2}}\right) .
$$

Without lost of generality, we may then suppose that

$$
\frac{1}{\beta_{1}}, \frac{1}{\beta_{2}} \leq \frac{1}{\alpha_{1}}=\frac{1}{\alpha_{2}} .
$$

From Fact 1 and Fact 2, one deduces that $\overline{\operatorname{conv}}\left(a_{1}, a_{1}^{\prime}, a_{2}, a_{2}^{\prime}\right)$ is a face of $Q$ and $\overline{\operatorname{conv}}\left(b_{2}, a_{1}, a_{1}^{\prime}\right)$ and $\overline{\operatorname{conv}}\left(b_{1}, a_{2}, a_{2}^{\prime}\right)$ are part of faces of $Q$. So $a_{1}$ and $a_{2}$ are the intersection of exactly 3 faces of $Q$. Let $L=Q^{s(Q)}$. Then $L$ is a polytope. Let $f$ be the vertex of $L$ corresponding to the face $\overline{\operatorname{conv}}\left(a_{1}, a_{2}, b_{1}, b_{2}\right)$ of $Q$. Using the correspondence between the face lattices of a polytope and its dual (see, for example, [15, Chap. 5.1] or [9, Chap. 3.4]), we see that there are exactly 4 faces, say, $F_{1}, F_{2}, G_{1}, G_{2}$ of $L$ containing $f$, corresponding to $a_{1}, a_{2}, b_{1}, b_{2}$. Moreover, $F_{1}$ and $F_{2}$ are triangles (because, $a_{1}$ and $a_{2}$ are the intersection of exactly 3 faces of $Q$ ). Let $\ell$ be the intersection of the supporting hyperplanes of $G_{1}$ and $G_{2}$, and let $\theta \in \mathbb{S}^{n-1}$ be the direction of $\ell$. We define the shadow movement of $L$ with basis $f$ and direction $\theta$. By Lemma 5 for some $c>0$, this shadow movement is volume affine on $[-c, c]$. We conclude using Lemmas 2 and 4.

Subcase 1.ii: The point $m$ is in the interior of an edge of $Q$. We may suppose that $m$ is an interior point of an edge $\left[a_{1}, b_{1}\right]$ of $H_{1} \cap Q$ and is an extreme point of $H_{2} \cap Q$. Let $a_{2} \neq b_{2}$ such that $\left[a_{2}, m\right]$ and $\left[m, b_{2}\right]$ are edges of $H_{2} \cap Q$. Then the two faces of $Q$ containing the edge $\left[a_{1}, b_{1}\right]$ of $Q$ are the triangles $\left(a_{1}, b_{1}, a_{2}\right)$ and $\left(a_{1}, b_{1}, b_{2}\right)$. Observe that by $a_{1}$ cannot pass more than two non-triangular faces, and that these non-triangular faces must have $\left[a_{1}^{\prime}, a_{1}\right]$ as an edge, where, as before, we denote by $a_{1}^{\prime}$ the vertex of $H_{1} \cap Q$ different from $b_{1}$ such that $\left[a_{1}, a_{1}^{\prime}\right]$ is an edge of $H_{1} \cap Q$. Define a shadow movement $Q_{t}$ with basis $a_{1}$ and direction $\theta$ parallel to the line supporting $\left[a_{1}, a_{1}^{\prime}\right]$. Then, from Lemma 5, for some $c>0, Q_{t}$ is volume affine and $Q_{t} \in \mathcal{C}_{N}$ on $[-c, c]$. We conclude again using Lemmas 2 and 4 .

Subcase 1.iii: The point $m$ is a vertex of $Q$. If $m$ is a vertex of $Q$, then all the faces containing $m$ are triangles. We move $m$ on the segment $[m, p]$ and, from Lemma 5, we get a shadow movement $Q_{t}$ such that $t \mapsto\left|Q_{t}\right|$ is affine on $[-c, c]$ for some $c>0$, and $Q_{t} \in \mathcal{C}_{N}$. We conclude as above. 
Case 2: $H_{1} \cap H_{2} \cap Q$ is one point $m$ It is easy to see that if $Q$ is not a double cone nor a cone then $m$ is a vertex of $H_{1} \cap Q$ and $H_{2} \cap Q$. Let $a_{i}, b_{i} \in Q \cap H_{i}$ be vertices of $Q \cap H_{i}$ such that $\left[a_{i}, m\right]$ and $\left[b_{i}, m\right]$ are edges of $Q \cap H_{i}$ (and thus of $Q$ ), $i=1,2$. Then the faces of $Q$ containing $m$ are the two triangles $\left(a_{1}, m, a_{2}\right),\left(b_{1}, m, b_{2}\right)$ and the two other faces $Q \cap H_{i}, i=1,2$. We define the affine shadow movement with basis $m$ in the direction of the line $H_{1} \cap H_{2}$ and we conclude as above.

Case 3: $H_{1} \cap H_{2} \cap Q=\emptyset \quad$ In this case $K_{i}=H_{i} \cap Q$ are faces of $Q$. We call the other faces of $Q$ side faces. It is clear that the side faces of $Q$ can be only triangles or quadrilaterals: any side face of $Q$ is either the convex hull of an edge of $K_{1}$ and an edge of $K_{2}$ or the convex hull of an edge of $K_{1}$ and a vertex of $K_{2}$, or the convex hull of an edge of $K_{2}$ and a vertex of $K_{1}$. We distinguish two cases.

Subcase 3.i: $Q$ has a side face $F$ which is a triangle. Without loss of generality, we may suppose that $F=\overline{\operatorname{conv}}\left(u_{1}, v_{1}, v_{2}\right)$, where $\left[u_{1}, v_{1}\right]$ is an edge of $K_{1}$ and $v_{2}$ is a vertex of $K_{2}$. If $K_{2}=\left\{v_{2}\right\}$ we conclude applying Lemma 1. Otherwise, among the faces of $Q$ containing $v_{1}$, those which have $\left[u_{1}, v_{1}\right]$ as an edge are $F$ and $K_{1}$. If there is a an other side face $G$ of $Q$ containing $v_{1}$ which is a quadrilateral, it must be of the form $G=\overline{\operatorname{conv}}\left(v_{1}, a_{2}, b_{2}, w_{1}\right)$, where $\left[v_{1}, w_{1}\right]$ is the other edge of $K$ containing $v_{1}$, and $\left[a_{2}, b_{2}\right]$ is an edge of $K_{2}$ (with possibly $a_{2}=v_{2}$ ). So there are at most 2 faces of $Q$ passing through $v_{1}$ which are not triangles. We are thus in position to apply Lemma 5 . With the notation of this lemma, we define the volume affine shadow movement $K_{t}$ with basis $v_{1}$ and in the direction of $\left[v_{1}, w_{1}\right]$. By Lemma 5 there exists $c>0$ such that $\left(K_{t}\right)$ is volume affine on $[-c, c]$. We conclude using Lemmas 4 and 2 .

Subcase 3.ii: There is no triangle among the side faces. Thus all the side faces of $Q$ are quadrilateral. Because of the special configuration of $Q$, we see that each vertex of $Q$ belongs to exactly three faces, two among them are quadrilateral side faces. Thus $Q$ is a simple polytope, and its polar body $L:=Q^{s(Q)}$ is a simplicial polytope (all its faces are triangles). The polytope $L$ has two special vertices $k_{1}$ and $k_{2}$ (dual to faces $H_{1} \cap Q$ and $H_{2} \cap Q$ of $Q$ ) such that all the faces of $L$ contain either $k_{1}$ or $k_{2}$. Through all the other vertices of $L$ pass exactly four triangular faces of $L$, two of them containing $k_{1}$ and the two others $k_{2}$. Let $r \neq k_{1}, k_{2}$ be a vertex of $L$. Fix any $\theta \in \mathbb{S}^{2}$ and define a shadow movement $\left(L_{t}\right)$ based on $r$ with direction $\theta$. We also note that $t \mapsto\left|L_{t}\right|$ is affine and $L_{t}^{s(L)} \in \mathcal{C}_{N}$, for small enough $|t|$. We use Lemmas 2 and 4 to conclude this case.

\subsection{Proof of the Centrally Symmetric Case}

The proof is exactly the same as the proof of the general case, except that the movements will now keep the symmetry. Moreover, as mentioned in Remark 1 after Theorem 1, there are only two cases: either $H_{1} \cap H_{2} \cap Q$ is a segment or it is empty, in which case the planes are parallel. Case 2 of the preceding discussion does not occur. Let us show how the arguments may be adapted to the symmetric case in Case 3, when $H_{1}$ and $H_{2}$ are parallel. By an adaptation of Lemma 4 there is a polyhedron $Q$ with minimal volume product in the set $\left\{K \in \mathcal{D}_{2 N}: K=-K\right\}$. We reduce to the case when $Q$ is formed as follows: $Q=\overline{\operatorname{conv}}\left(M+e_{3},-M-e_{3}\right)$ where $M$ is a polygon 
in the hyperplane $\left\{x_{3}=0\right\}$ with $N$ vertices. We shall show that

$$
\mathcal{P}(Q)=\mathcal{P}\left(Q_{1}\right)=\mathcal{P}\left(Q_{2}\right)=\frac{32}{3},
$$

where $Q_{i}=\overline{\operatorname{conv}}\left(Q_{i}+e_{3},-Q_{i}-e_{3}\right)$ with $Q_{1}$ a triangle and $Q_{2}$ a parallelogram. Actually $Q_{1}$ is affinely equivalent to $B_{1}^{3}$ and $Q_{2}$ to $B_{\infty}^{3}$, which are the two, non affinely equivalent, conjectured mimima for the volume product of centrally symmetric bodies in $\mathbb{R}^{3}$. We begin as in the proof of Theorem 1 , but we apply now shadow systems which keep the central symmetry of $Q$.

Subcase i: There is a triangle $F$ among the side faces of $Q$. Since $Q$ is centrally symmetric, the face $-F$ symmetric of $F$ is also a triangle. One may suppose that $F=\overline{\operatorname{conv}}\left(u+e_{3},-v-e_{3},-w-e_{3}\right)$ where $u, v, w$ are different extreme points of $Q$ and $[v, w]$ is an edge of $Q$. Consider now the shadow system constructed as follows. Define

$$
Q_{t}=\overline{\operatorname{conv}}\left(\mathcal{E}(Q) \backslash\left\{v+e_{3},-v-e_{3}\right\}, v+e_{3}+t \theta,-v-e_{3}-t \theta\right),
$$

for $t \in \mathbb{R}$ and $\theta \in \mathbb{S}^{2}$ is the direction of the edge [ $\left.v, w\right]$. We notice that at most two faces of $Q$ which are not triangles can contain $v+e_{3}$ (the same is true for $-v-e_{3}$ ), and we may apply Lemma 5. Thus, for some $c>0, t \mapsto\left|Q_{t}\right|$ is affine for $t \in[-c, c]$. Applying the minimality of $\mathcal{P}(Q)$ we get $\mathcal{P}(Q)=\mathcal{P}\left(Q_{0}\right)=\mathcal{P}\left(Q_{t}\right)$; it follows from Proposition 1 that $Q_{t}$ is an affine image of $Q$, say $Q_{t}=A_{t}(Q)$, with $A_{t}$ of the type described in Proposition 1. Thus $Q$ is a double cone with apices $v+e_{3},-v-e_{3}$ and we conclude the proof applying Lemma 2.

Subcase ii: There is no triangle among the side faces of $Q$. Then they are all quadrangles. We pass to the polar body $L=Q^{\circ}$, which is of course also centrally symmetric, and has all the properties mentioned in Subcase 3.ii, in the proof of the non-symmetric case. We modify it with a "symmetric shadow movement" $L_{t}$, moving two of its vertices $v$ and $-v$, along one edge. As before, by minimality this shadow movement must satisfy $L_{t}=A_{t}(L)$ for $t \in[-c, c]$, for some $c>0$, where $A_{t}$ is an affine isomorphism of $\mathbb{R}^{3}$. It is easy to see that this can only happen when $L$ is a double cone with apices $-v$ and $v$. So that, by the minimality of the volume product, its basis must be a quadrilateral. So that $L$ is affinely isomorphic to $B_{1}^{3}$ and $P=L^{\circ}$ is affinely isomorphic to $B_{\infty}^{3}$.

Acknowledgements The first author was supported in part by the Agence Nationale de la Recherche, project GeMeCoD (ANR 2011 BS01 007 01) and the third author by U.S. National Science Foundation grant DMS-1101636 and Université Paris-Est Marne-la-Vallée.

\section{References}

1. Barthe, F., Fradelizi, M.: The volume product of convex bodies with many hyperplane symmetries. Am. J. Math., to appear

2. Bourgain, J., Milman, V.D.: New volume ratio properties for convex symmetric bodies in $\mathbb{R}^{n}$. Invent. Math. 88, 319-340 (1987)

3. Campi, S., Gronchi, P.: On volume product inequalities for convex sets. Proc. Am. Math. Soc. 134(8), 2393-2402 (2006) 
4. Fradelizi, M., Gordon, Y., Meyer, M., Reisner, S.: The case of equality for an inverse Santalo functional inequality. Adv. Geom. 10, 621-630 (2010)

5. Giannopoulos, A., Paouris, G., Vritsiou, B.-H.: The isotropic position and the reverse Santalo inequality. Isr. J. Math., to appear

6. Gordon, Y., Meyer, M.: On the minima of the functional Mahler product. Houst. J. Math., to appear

7. Gordon, Y., Meyer, M., Reisner, S.: Zonoids with minimal volume-product-a new proof. Proc. Am. Math. Soc. 104, 273-276 (1988)

8. Gruber, P.M.: Approximation of convex bodies. In: Gruber, P.M., Wills, J.M. (eds.) Convexity and Its Applications, pp. 131-162. Birkhäuser, Basel (1983)

9. Grünbaum, B.: Convex Polytopes. Graduate Texts in Mathematics, vol. 221. Springer, Berlin (2003)

10. Hansen, A., Lima, A.: The structure of finite-dimensional Banach spaces with the 3.2 intersection property. Acta Math. 146(1-2), 1-23 (1981)

11. Kim, J., Reisner, S.: Local minimality of the volume-product at the simplex. Mathematika 57, 121134 (2011)

12. Kuperberg, G.: From the Mahler conjecture to Gauss linking integrals. Geom. Funct. Anal. 18, 870892 (2008)

13. Mahler, K.: Ein Minimalproblem für konvexe Polygone. Mathematica (Zutphen) B 7, 118-127 (1939)

14. Mahler, K.: Ein Überträgungsprinzip für konvexe Körper. Čas. Pěst. Math. Fys. 68, 93-102 (1939)

15. Matousek, J.: Lectures on Discrete Geometry. Graduate Texts in Mathematics, vol. 212. Springer, Berlin (2002)

16. Meyer, M.: Une caractérisation volumique de certains espaces normés. Isr. J. Math. 55, 317-326 (1986)

17. Meyer, M.: Convex bodies with minimal volume product in $\mathbb{R}^{2}$. Monatshefte Math. 112, 297-301 (1991)

18. Meyer, M., Pajor, A.: On Santaló inequality. In: Geometric Aspects of Functional Analysis (1987-88). Lecture Notes in Math., vol. 1376, pp. 261-263. Springer, Berlin (1989)

19. Meyer, M., Reisner, S.: Inequalities involving integrals of polar-conjugate concave functions. Monatshefte Math. 125, 219-227 (1998)

20. Meyer, M., Reisner, S.: Shadow systems and volumes of polar convex bodies. Mathematika 53(1), 129-148 (2006)

21. Nazarov, F.: The Hörmander proof of the Bourgain-Milman theorem. Preprint (2009)

22. Nazarov, F., Petrov, F., Ryabogin, D., Zvavitch, A.: A remark on the Mahler conjecture: local minimality of the unit cube. Duke Math. J. 154(3), 419-430 (2010)

23. Petty, C.M.: Affine isoperimetric problems. Ann. N.Y. Acad. Sci. 440, 113-127 (1985)

24. Pisier, G.: The Volume of Convex Bodies and Banach Space Geometry. Cambridge Tracts in Mathematics, vol. 94. Cambridge University Press, Cambridge (1989)

25. Reisner, S.: Zonoids with minimal volume-product. Math. Z. 192, 339-346 (1986)

26. Reisner, S.: Minimal volume product in Banach spaces with a 1-unconditional basis. J. Lond. Math. Soc. 36, 126-136 (1987)

27. Reisner, S., Schütt, C., Werner, E.M.: Mahler's conjecture and curvature. Int. Math. Res. Not. (2011). doi:10.1093/imrn/rnr003

28. Rogers, C.A., Shephard, G.C.: Some external problems for convex bodies. Mathematika 5, 93-102 (1958)

29. Santaló, L.A.: Un invariante afin para los cuerpos convexos del espacio de $n$ dimensiones. Port. Math. 8, 155-161 (1949)

30. Saint Raymond, J.: Sur le volume des corps convexes symétriques. Séminaire D'Initiation à L'Analyse, 1980-81, Université Paris VI (1981)

31. Schneider, R.: Convex Bodies: The Brunn-Minkowski Theory. Cambridge University Press, Cambridge (1993)

32. Schneider, R., Wieacker, J.A.: Approximation of convex bodies by polytopes. Bull. Lond. Math. Soc. 13, 149-156 (1981) 